\title{
Atomic Geometry and Energetics of Native Defects in Cubic Boron Nitride
}

\author{
W. Orellana and H. Chacham \\ Departamento de Física, Universidade Federal de Minas Gerais, \\ CP 702, 30161-970, Belo Horizonte, MG, Brazil
}

Received February 8, 1999

\begin{abstract}
We use first-principles calculations to investigate the electronic structure, atomic geometries and formation energies of point vacancies $\left(V_{B}, V_{N}\right)$, antisites $\left(B_{N}, N_{B}\right)$ and interstitials $\left(B_{i}, N_{i}\right)$ in cubic boron nitride $(\mathrm{c}-\mathrm{BN})$. We find that nitrogen and boron vacancies exhibit the lowest formation energies in nonstoichiometric c-BN for $p$-type and $n$-type conditions, respectively, showing intrinsic donor $\left(\mathrm{V}_{B}\right)$ and acceptor $\left(\mathrm{V}_{N}\right)$ characters. The equilibrium geometries show large outward breathing relaxations for both vacancies and for $\mathrm{B}_{N}$, with slight $\mathrm{J}$ ahn-Teller distortions from $T_{d}$ symmetry. For $\mathrm{N}_{B}$ we find an off-center distortion inducing a negative- $U$ behavior in this center. For both interstitial centers we find stable configurations in which an atomic pair occupies a nitrogen site in the lattice, $\mathrm{N}-\mathrm{N}$ for $\mathrm{N}_{i}$ and $\mathrm{N}-\mathrm{B}$ for $\mathrm{B}_{i}$. These systems are stable for the different charge states investigated.
\end{abstract}

Cubic boron nitride (c-BN) has recently emerged as an important material for semiconducting industry due to its interesting properties which make it ideal for ultraviolet optoelectronic and high-temperature devices. Despite the recent synthesis of high-quality c$\mathrm{BN}$ single crystals[1] $\Gamma$ the high concentration of crystalline defects remains the central problem for experimental research in $\mathrm{c}-\mathrm{BN}$. Conductivities related to defects or impurities have been observed in doped c-BN.[2Г 3] In fact $\Gamma$ electron paramagnetic resonance (EPR) measurements[4Г5] for different allotropic forms of boron nitride ascribe to the nitrogen vacancy the dominant paramagnetic defect in this material.

There are few theoretical studies related to native defects in c-BN. Vacancies and/or antisites have been studied with pseudopotentialI[6] tight-binding $\mathbb{1}[7]$ cluster[8] and LAPW[9] methods. HoweverT in most of these calculations the atomic relaxation is restricted to the first-neighbor atoms[8Г 9] or simply not considered[7].

In this work we present a comparative study of the electronic structure and energetics of native defecs in c-BNT using first-principles total energy calculations. For each defect $\Gamma$ we have determined the fully-relaxed atomic geometry in all relevant charge states. Our calculations are based on the densityfunctional theoryI[10] using the supercell approach with
64 atoms per cell $\Gamma$ a plane-wave basis set with a kinetic energy cutoff of $60 \mathrm{Ry}$ T and norm-conserved soft Troullier-Martins pseudopotentials.[11] For the exchange-correlation functional $\Gamma$ we use the generalized gradient approximation (GGA).[12] The integration over the Brillouin zone is performed with one special k-point (the $\Gamma$ point). The geometry optimization is obtained by calculating Hellmann-Feynman forces on the nuclei. The atomic positions are supposed to converge when the forces on the atoms are less than $0.05 \mathrm{eV} / \AA$.

The formation energies of native defects in c- $\mathrm{BN}$ are calculated as a function of the chemical potentials of nitrogen and boron. For charged systemsTthe formation energy also depends on the position of the Fermi level. Thus $\Gamma$ for a defect in the charge state $q$ T the formation energy is given by [13]

$$
E_{\text {form }}(q)=E_{t}(q)-n_{N} \mu_{N}-n_{B} \mu_{B}+q\left(\mu_{e}+E_{\imath}\right)
$$

where $E_{t}$ is the total energy of the defect derived from the supercell calculation $\Gamma n_{N}\left(n_{B}\right)$ is the number of $\mathrm{N}$ (B) atoms in the supercell $\Gamma$ and $\mu_{N}\left(\mu_{B}\right)$ is the corresponding chemical potential $\Gamma \mu_{e}$ is the electronic chemical potential or the position of the Fermi level relative to the valence band edge $\Gamma$ and $E_{v}$ is the energy of the top of the valence band for bulk c-BN. The atomic chemical potentials can vary over a range given by the heat 
of formation of c-BNTwhich we calculated as $3.0 \mathrm{eV}$. Additionally they are constrained by the equilibrium condition $\Gamma \mu_{N}+\mu_{B}=\mu_{c B N(b u l k)}$. Upper bounds for $\mu_{N}$ and $\mu_{B}$ are the precipitation limits on bulk phases $\Gamma$ $\mu_{N}<\mu_{N(b u l k)}$ and $\mu_{B}<\mu_{B(b u l k)}$ Twhich are calculated from solid nitrogen $\left(\alpha-\mathrm{N}_{2}\right)$ and metallic boron $(\alpha-\mathrm{B}) \Gamma$ respectively.

Our results for the equilibrium geometries and relaxation energies of both vacancies in different charge states are summarized in Table 1 . The vacancies in the neutral charge state show large outward breathing relaxations of the nearest-neighbor atoms Twith slight distortions from the $T_{d}$ symmetry. For neutral $\mathrm{V}_{B}\left(\mathrm{~V}_{N}\right) \Gamma$ the distance from the center of the vacancy to its $\mathrm{N}$ (B) first-neighbor atoms is found to be $10.5 \%(7.7 \%)$ larger than in the unrelaxed system $\Gamma$ while the distance between the first and second neighbors is $4.3 \%(2.9 \%)$ shorter. The relaxation energies $\Gamma$ calculated from the difference between the relaxed and the unrelaxed systems Tshow that $\mathrm{V}_{N}$ suffers a more drastic relaxation with changes in the charge states as compared with $\mathrm{V}_{B}$. This suggests a stronger interaction among the dangling bonds on the neighboring atoms of $V_{N}$ than the corresponding for $\mathrm{V}_{B}$.

Table 1. Change in the atomic geometry with respect to the unrelaxed system (in percent) for boron and nitrogen vacancies in c-BN. $\Delta d_{1}$ is the change in the distance between the vacancy center and the first-neighbor atoms. $\Delta d_{2}$ is the change in the distance between first and second-neighbor atoms. $E_{r}$ is the relaxation energy $\mathrm{Tin} \mathrm{eV}$.

\begin{tabular}{ccccccc} 
& \multicolumn{3}{c}{$\mathrm{V}_{N}$} & \multicolumn{3}{c}{$\mathrm{V}_{B}$} \\
Charge & $\Delta d_{1}$ & $\Delta d_{2}$ & $E_{r}$ & $\Delta d_{1}$ & $\Delta d_{2}$ & $E_{r}$ \\
\hline $3+$ & 19.8 & -5.7 & 2.79 & & & \\
$2+$ & 15.4 & -4.8 & 1.79 & & & \\
$1+$ & 7.6 & -2.9 & 0.72 & 10.8 & -4.0 & 1.83 \\
0 & 7.7 & -2.9 & 1.19 & 10.5 & -4.3 & 2.14 \\
$1-$ & & & & 10.4 & -4.9 & 2.29 \\
$2-$ & & & & 10.3 & -5.8 & 2.57 \\
$3-$ & & & & 10.3 & -6.5 & 2.70 \\
\hline
\end{tabular}

The electronic structure of the native defects in c$\mathrm{BN}$ is presented schematically in Fig. 1. In the figure $\mathrm{T}$ we show the positions of the defect-induced one-electron states in the band gap $\Gamma$ for the neutral vacancies $\Gamma$ antisites and interstitials. For $\mathrm{V}_{N}^{0}$ Twe find a fully-occupied s-like $a_{1}$ state in the band gap close to the valence band edge $\Gamma$ and a doublet state slightly split into singlets $\Gamma l y-$ ing as an e-like resonance in the bottom of the conduction band. Because this resonance is singly occupied $\Gamma$ the neutral charge state is unstable $\Gamma$ and $\mathrm{V}_{N}$ only exists in donor states. For $V_{B}^{0}$ Twe find a p-like $t_{2}$ state in the lower part of the band gap $\Gamma$ which also shows a slightly split into singlet states. This $t_{2}$ state is half occupied $\Gamma$ thus acting as a triple acceptor. The small splitting of the partially filled degenerate levels indicates a very small Jahn-Teller (JT) effect in the neutral vacancies. The difference in energy between split levels is less than $10 \mathrm{meV}$.

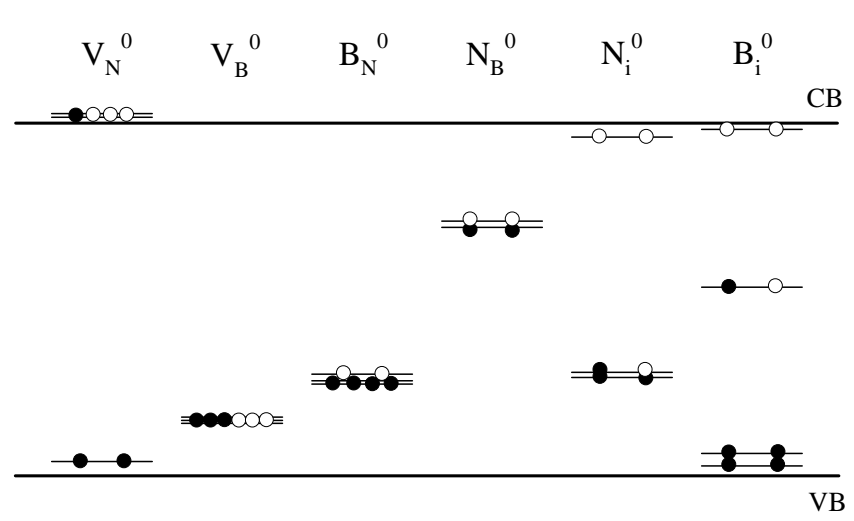

Figure 1. Schematic representation of the position of oneelectron levels in the band gap induced by native defects in $\mathrm{c}-\mathrm{BN}$ in neutral charge states. The filled circles indicate electrons and the open circles indicate holes.

The equilibrium geometry for $\mathrm{B}_{N}^{0}$ shows an outward breathing relaxation of the neighboring $B$ atoms Twith a small distortion of the $B$ antisite along the [100] direction (about $0.03 \AA$ from the $\mathrm{N}$ site). The distance between $\mathrm{B}_{N}$ and its first-neighbor atoms is $5.2 \%$ larger than in the unrelaxed system $\Gamma$ while the distances between first and second neighbors are $0.2 \%$ shorter. No distortion along the [111] direction has been detected. We observe only small distortions of the $\mathrm{B}$ antisite for other charge states investigated. The electronic structure for $\mathrm{B}_{N}^{0}$ (Fig.1) shows a p-like $t_{2}$ state in the gap $\Gamma$ occupied with four electrons. This state also splits into singlet statesT due to the small JT distortion.

For $\mathrm{N}_{B}^{0} \Gamma$ we observe an off-center distortion of the $\mathrm{N}$ antisite along the [100] direction $\Gamma$ with a minimum at $0.35 \AA$ from the $\mathrm{B}$-site. The $\mathrm{N}$ antisite binds to two neighboring $\mathrm{N}$ atoms $\mathrm{f}$ forming a bridge structure with equal N-N bond lengths of $1.41 \AA$ Tand an angle of $122^{\circ}$ between the bonds. For $\mathrm{N}_{B}$ in negative charge states $\Gamma$ we also find the same distortions which are characterized by large lattice relaxations. However $\Gamma$ for positive charge states we observe a breathing relaxation $\Gamma$ preserving the $T_{d}$ symmetry. In Table $2 \Gamma$ we summarize our results for the stable geometries and relaxation energies of the $\mathrm{N}$ antisite for different charge states. 
Table II. Stable geometry for the nitrogen antisite in cBN. $d$ is the distance between the antisite and their first-neighbor $\mathrm{N}$ atoms $\Gamma$ and $\theta$ is the angle between them. $E_{r}$ is the relaxation energy.

\begin{tabular}{ccccc} 
Charge & Symmetry & $d[\AA]$ & $\theta\left[^{\circ}\right]$ & $E_{r}[\mathrm{eV}]$ \\
\hline $2+$ & $T_{d}$ & 1.528 & 109.5 & 0.51 \\
$1+$ & $T_{d}$ & 1.628 & 109.5 & 0.60 \\
0 & $C_{2 v}$ & 1.408 & 122.0 & 2.41 \\
$1-$ & $C_{2 v}$ & 1.412 & 122.7 & 3.92 \\
$2-$ & $C_{2 v}$ & 1.425 & 123.7 & 4.96 \\
\hline
\end{tabular}

Considering that previous calculations[14] have reported an off-center stable geometry for $\mathrm{N}_{B}^{0}$ with $C_{3 v}$ symmetry we have calculated the total energy surface as a function of the nitrogen displacement along the [100] and [111] directions. This is done by placing the $\mathrm{N}$ antisite in various positions along these directions Tallowing the neighbor atoms to relax. These calculation are performed by considering four special $\mathbf{k}$-points[15] instead the $\Gamma$ point of our early calculation $\Gamma$ in order to ensure the convergence with respect to the Brillouin zone integration. Our results are shown in Fig.2 Twhere we see that the stable configuration has the $\mathrm{N}$ atom displaced along the [100] direction with respect to the substitutional site lowering the total energy by about $0.5 \mathrm{eV}$. No distortion along the [111] direction has been detected. This result agrees with our calculation with one $\mathbf{k}$-point. We also note that the $\mathrm{N}$ antisite can occupy a metastable position with $T_{d}$ symmetry. We attribute the discrepancy between our results and the calculation of Ref. 14 to the small supercell here used (16 atoms) implying in restrictions on the atomic relaxation.

The gap levels for $\mathrm{N}_{B}^{0}$ shown in Fig.1 correspond to the off-center configuration with $C_{2 v}$ symmetry. We find a fully-occupied s-like $a_{1}$ state in the upper part of the band gap $\Gamma$ close to an empty $a_{1}$ state. The difference in energy between these states is $0.07 \mathrm{eV}$. The empty $a_{1}$ state originates from the split of a $t_{2}$ state localized close the bottom of the conduction band for the defect in $T_{d}$ symmetry. The empty and occupied $a_{1}$ states move toward one another while the $\mathrm{N}$ antisite moves along the [100] direction to its stable configuration.

For the self interstitial defects in c- $\mathrm{BN}\left(\mathrm{N}_{i}\right.$ and $\left.\mathrm{B}_{i}\right) \Gamma$ we consider different interstitial sites in the lattice as a starting positions for $\mathrm{N}$ and $\mathrm{B}$ atoms in the molecular dynamic calculation. These sites are: bonding (between a B-N bond) Tantibonding (opposite to the bonding site with respect to a lattice atom) Ttetrahedral and hexagonal. This procedure is necessary in order to avoid possible metastable positions for the interstitial atoms. Our results for the minimum energy configuration of $\mathrm{N}_{i}^{0}$ show the formation of a N-N pair occuping a nitrogen site in the lattice $\Gamma$ where each atom of the pair binds to two neighboring $\mathrm{B}$ atoms. The $\mathrm{N}-\mathrm{N}$ bond distance is found in $1.28 \AA$ Twhich is $16 \%$ larger than that of the $\mathrm{N}_{2}$ molecule $(1.1 \AA)$. The bond distance between one nitrogen of the pair and the boron first neighbors is $1.42 \AA$ A with a B-N-B angle of $141^{\circ}$. This same configuration is also obtained for the $\mathrm{N}_{i}$ center in other charge states. The electronic structure of $\mathrm{N}_{i}^{0}$ (Fig.1) shows two midgap singlet states Tone fully occupied and the other one half occupied $\Gamma$ and a empty singlet state close to the bottom of the conduction band. The midgap levels correspond to two $\mathrm{p}_{z}$-like dangling bonds located at each $\mathrm{N}$ atom of the pairTwhich are oriented normal to the plane formed by the B-N-B atoms.

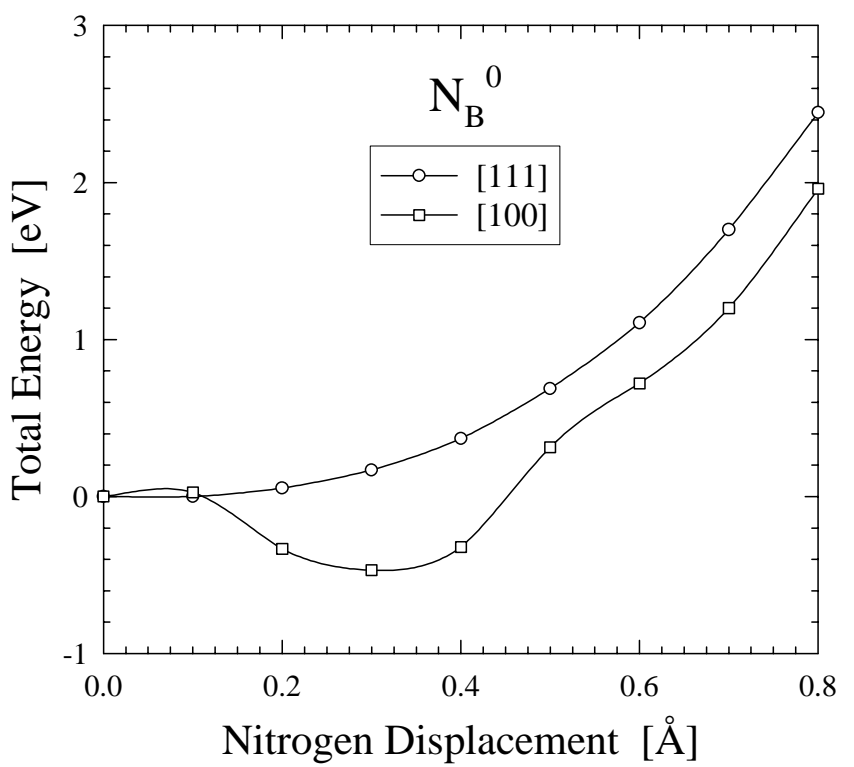

Figure 2. Total energy as a function of the nitrogen displacement along the [111] and [100] directions for the neutral $\mathrm{N}_{B}$ in $c-B N$. The zero energy refers to the total energy with nitrogen at the substitutional site.

For the $\mathrm{B}_{i}^{0}$ defect we find a minimum energy configuration in which a N-B pair substitutes a nitrogen atom in the lattice. The N-B bond distance is $1.32 \AA \Gamma$ which is $15 \%$ shorter than the N-B bond of bulk c-BN. The nitrogen of the $\mathrm{N}-\mathrm{B}$ pair binds to two neighboring $\mathrm{B}$ atoms $\mathrm{Twith}$ bond distance of $1.42 \AA$ and $\mathrm{B}-\mathrm{N}-\mathrm{B}$ angle of $140^{\circ}$. The boron of the pair binds to the other two neighboring $B$ atoms Twith bond distance of $1.52 \AA$ and B-B-B angle of $141^{\circ}$. This configuration is stable for different charge states. The electronic structure of $\mathrm{B}_{i}^{0}$ (Fig.1) shows a half-ocuppied singlet states in the middle of the gap and two fully-occupied singlet states close to the edge of the valence band. We also find a empty singlet state close to the bottom of the conduction band. Similarly to the $\mathrm{N}_{i}$ center $\Gamma$ the midgap 
singlet state of the $\mathrm{B}_{i}$ center correspond to a $\mathrm{p}_{z}$-like dangling bond located at the $\mathrm{N}$ atom normal to the plane formed by the B-N-B atoms. In this configuration the $\mathrm{N}$ atom is bound to three $\mathrm{B}$ atoms in a $s p^{2}$ hybridization showing a $\mathrm{p}_{z}$-like dangling bond. This structure resembles the hexagonal phase of boron nitride. However $\Gamma$ the boron of the $\mathrm{N}-\mathrm{B}$ pair is bound to three neighboring $\mathrm{B}$ atoms forming metallic bonds.

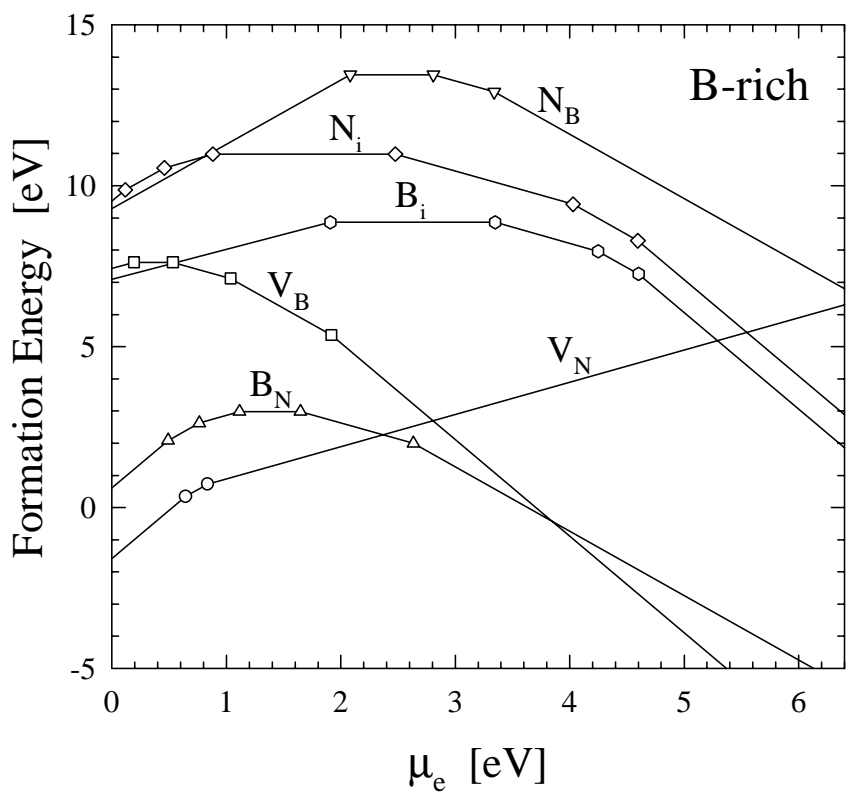

Figure 3. Formation energies as a function of the electronic chemical potential (Fermi level), of native defects in c-BN for nitrogen-rich conditions, $\mu_{B}=\mu_{B(b u l k)}$. The slopes of the line segments characterize to defects charge states, and the symbols characterize transition states.

In Fig. 3Twe show our results for the formation energies of native defects as a function of the Fermi energy $\left(\mu_{e}\right)$ for boron-rich conditions. In this figure Tthe slopes of the line segments correspond to the charge states of the defects. Changes in the slopes indicate transition states $\Gamma$ which are represented by symbols. We observe that for $p$-type conditions (Fermi energy close to the top of the valence band) $\Gamma V_{N}^{3+}$ has the lowest formation energies among the native defects and can be considered as the dominant defect in $p$-type c-BN. On the other hand $\Gamma$ for $n$-type conditions (Fermi energy close to the bottom of the conduction band) $\Gamma V_{B}^{3-}$ exhibits the lowest formation energy $\Gamma$ and hence dominantes in $n$-type c-BN. We also note that the boron antisite exhibits low formation energies in the entire range of the band gap $\Gamma$ which are comparable to those of the vacancies. In fact $\Gamma \mathrm{B}_{N}$ has the lowest formation energy for $2.4 \mathrm{eV}<\mu_{e}<3.8 \mathrm{eV}$ Tand thus can be considered a dominant defect for semi-insulating c-BN. Another defects that show low formation energies in $n$-type conditions are the interstitials. $\mathrm{N}_{i}^{3-}$ and $\mathrm{B}_{i}^{3-}$ exhibit low formation energies which can be readily incorporated in $n$-type c-BN. Because the relatively low formation energies found for $\mathrm{V}_{N}^{3+}$ in $p$-type condition and for $\mathrm{V}_{B}^{3-}$ for $n$-type condition $\Gamma$ we believe they could act as a compensation centers in doped c-BN.

We now turn our attention to important subjects derived from our calculations. We find that the nitrogen vacancy in $n$-type c-BN shows high formation energies $(>6 \mathrm{eV})$. Therefore $\Gamma$ the observed $n$-type conductivity[3] usually associated to the nitrogen vacancy would imply either in a nonequilibrium incorporation of this defect or in the incorporation of a donorlike impurity with lower formation energy. According to our equilibrium calculation $\Gamma$ the nitrogen vacancy should not be responsible for the $n$-type conductivity. The same picture has also been suggested fo $V_{N}$ in GaN.[16]

AdditionallyCour results show that $\mathrm{N}_{B}^{+}$is never stable which characterizes a negative- $U$ behavior for this defect. From the $(2+/+)$ and $(+/ 0)$ transition states we obtain $\mathrm{U}=\mathrm{E}(+/ 0)-\mathrm{E}(2+/+) \approx-0.7 \mathrm{eV}$. The origin of the negative- $U$ behavior is related to the offcenter distortion of the nitrogen antisite in the neutral charge state. The $(2+/ 0)$ transition located at $2.1 \mathrm{eV}$ from the top of the valence band $\Gamma$ is characterized by a large lattice relaxation $\Gamma$ with an energy gain by about $2 \mathrm{eV}$.

FinallyCEPR measurements have reported paramagnetic defects in bulk[4] and thin-film[5] boron nitride $\Gamma$ which are commonly associated to $V_{N}$ in neutral charge state. Our results show that $\mathrm{V}_{N}$ only exists in positive charge states $\Gamma$ supporting that $\mathrm{V}_{N}^{2+}$ Tinstead $\mathrm{V}_{N}^{0}$ Tshould be the paramagnetic center associated to the nitrogen vacancy in $c-B N$.

In summaryTwe have foundTbased on first-principles calculations $\Gamma$ that nitrogen and boron vacancies are the dominant defects in nonstoichiometric c-BN for $p$-type and $n$-type conditions $\Gamma$ respectively. The vacancies also show intrinsic donor $\left(\mathrm{V}_{N}\right)$ and acceptor $\left(\mathrm{V}_{B}\right)$ characters which makes them the main candidates for the experimentally observed dopant compensation. MoreoverTaccording to our calculations the high formation energies observed for $\mathrm{V}_{N}$ in $n$-type conditions exclude this vacancy as a source of $n$-type conductivity. For $\mathrm{N}_{B}$ we find a negative- $U$ behavior originates in an off-center distortion of the $\mathrm{N}$ antisite along [100] direction. Finally for the interstitial centers we find configurations 
in which the interstitial atom forms an atomic pair with a lattice atom occuping a nitrogen site $\mathrm{NN}-\mathrm{N}$ for $\mathrm{N}_{i}$ and $\mathrm{N}-\mathrm{B}$ for $\mathrm{B}_{i}$. These systems show midgap levels in the band gap and are stable for different charge states.

This work was supported by the Brazilian agencies FAPEMIG and CNPq. The calculations were performed at CENAPAD-MG/CO.

\section{References}

[1] T. Taniguchi and S. Yamaoka, Mat. Res. Soc. Symp. Proc. 472, 379 (1997).

[2] O. Mishima, J. Tanaka, S. Yamaoka and O. Fukunaga, Science 238, 181 (1987).

[3] O. Mishima, K. Era, J. Tanaka and S. Yamaoka, Appl. Phys. Lett. 53, 962 (1988).

[4] F. Zhang and G. Chen, Mat. Res. Soc. Symp. Proc., 242, 613 (1992).

[5] M. Fanciulli and T. D. Moustakas, Physica B 185, 228 (1993).

[6] Y. Bar-Yam, T. Lei, T. D. Moustakas, D. C. Allan, and M. P. Teter, Mat. Res. Soc. Symp. Proc. 242, 335 (1992).
[7] V. A. Gubanov, Z. W. Lu, M. Klein, and C. Y. Fong, Phys. Rev. B 53, 4377 (1996).

[8] P. Piquini, R. Mota, T. M. Schmidt and A. Fazzio, Phys. Rev. B 56, 3553 (1997).

[9] J. L. P. Castineira, J. R. Leite, L. M. R. Scolfaro, R. Enderlein, H. W. L. Alves and J. L. A. Alves, Radiat. Eff. Defect. S. 146, 49 (1998).

[10] P. Hohemberg and W. Kohn, Phys. Rev. 136, B 864 (1964); W. Kohn and L. J. Sham, Phys. Rev. 140, A1133 (1965).

[11] N. Troullier and J. L. Martins, Phys. Rev. B 43, 1993 (1991).

[12] J. Perdew, Phys. Rev. Lett. 55, 1665 (1985); J. P. Perdew and Y. Wang, Phys. Rev. B 33, 8800 (1986).

[13] S. B. Zhang and J. E. Northrup, Phys. Rev. Lett. 67, 2339 (1991).

[14] J. L. P. Castineira, J. R. Leite, L. M. R. Scolfaro, R. Enderlein, J. L. A. Alves and K. K. Bajaj, Proc. of ICPS-23, (World Scientific, Singapure, 1996), p. 2991,

[15] D. J. Chadi and M. L. Cohen, Phys. Rev. B 8, 5747 (1973).

[16] J. Neugenbauer and C. G. Van de Walle, Phys. Rev. B 50, 8067 (1994). 